\title{
Uptake of Gd-EOB-DTPA in Bone and Splenic Metastases from Hepatocellular Carcinoma: A Case Study
}

\author{
Zheng $\mathrm{L}^{1}$, Zhao $\mathrm{Y}^{1,2}$, Chen $\mathrm{W}^{1 *}$ and Tian $\mathrm{R}^{3}$ \\ ${ }^{1}$ Department of Radiology, Sichuan University, China \\ ${ }^{2}$ Department of Radiology, Central South University, \\ China \\ ${ }^{3}$ Department of Nuclear Medicine, Sichuan University, \\ China \\ *Corresponding author: Weixia Chen, Department \\ of Radiology, West China Hospital, Sichuan University, \\ Chengdu 610041, Sichuan Province, China
}

Received: December 29, 2016; Accepted: February 14, 2017; Published: February 17, 2017

\begin{abstract}
We present the case of bone and splenic metastases from hepatocellular carcinoma ( $\mathrm{HCC})$, which showed uptake of gadolinium-ethoxybenzyldiethylenetriaminepentaacetic acid (Gd-EOB-DTPA) on magnetic resonance imaging (MRI). To the best of our knowledge, this is the first case of bone and splenic metastases from HCC that demonstrated uptake of Gd-EOB-DTPA in the hepatobiliary phase.
\end{abstract}

Keywords: Gd-EOB-DTPA; HCC; Bone metastases; Splenic metastases

\section{Case Presentation}

A 65-year-old man presented with a 3-month history of dull pain on the right side of the chest. A liver tumour was found on routine abdominal magnetic resonance imaging (MRI), with elevated alpha-fetoprotein (AFP) at $498 \mathrm{ng} / \mathrm{ml}$ [normal range $<8 \mathrm{ng} / \mathrm{ml}$ ]. Gadolinium-ethoxybenzyl-diethylenetriaminepentaacetic acid (GdEOB-DTPA) enhanced MRI was then performed. Unenhanced MRI displayed an ill-defined heterogeneous mass, which was hyperintense on T2-weighted imaging (T2WI) and hypointense on T1-weighted imaging (T1WI), in the right lobe of the liver. After the injection of Gd-EOB-DTPA, the lesion showed heterogeneous enhancement in the arterial phase and washout in the portal venous and equilibrium phases. In the hepatobiliary phase, a large part of the lesion appeared hypointense, and multiple hyperintense nodules within the lesion were observed (Figure 1). Several hypointense satellite lesions with well-defined margin were observed only in the hepatobiliary phase.

An ill-defined mass with osteolytic destruction around the right seventh rib was noted; it had a similar enhancement pattern with the liver lesion and appeared hypointense in the hepatobiliary phase (Figure 1). Patchy, nodular lesions $(\leq 10 \mathrm{~mm})$ were observed in several thoracolumbar vertebrae (T5, T8, T9, T11, L1 and L2), in the right tenth rib and left ninth rib. These patchy, nodular lesions appeared hyperintense on T2WI, less clear on unenhanced T1WI, and showed markedly enhancement during the arterial and portal venous phases, with persistent enhancement in hepatobiliary phase (Figure 1). There were three splenic nodules measuring 7-10 $\mathrm{mm}$ in diameter, which were isointense on unenhanced T1WI and T2WI, but showed persistent enhancement from the arterial phase to hepatobiliary phase.

As part of the staging process, ${ }^{18} \mathrm{~F}$-fluorodeoxyglucose positron emission tomography/computed tomography $\left({ }^{18} \mathrm{~F}-\mathrm{FDGPET} / \mathrm{CT}\right)$ and bone scintigraphy (BS) were performed. On the PET scan, the liver mass showed ${ }^{18} \mathrm{~F}-\mathrm{FDG}$ uptake with a maximum standardized uptake value of 4.7. Only the lesion in the right seventh rib showed elevated ${ }^{18} \mathrm{~F}-\mathrm{FDG}$ uptake. The other bone lesions in thoracolumbar vertebrae (T5, T8, T9, T11, L1 and L2), in the right tenth rib and left ninth rib were small $(\leq 10 \mathrm{~mm})$ and didn't show elevated ${ }^{18} \mathrm{~F}-\mathrm{FDG}$ uptake (Figure 1). The spleen showed normal glucose uptake, without displaying the nodules that were found on the Gd-EOB-DTPAenhanced MRI. The BS images showed anomalous radioactive concentration in the right seventh and tenth ribs and T5 (Figure 2).

For this patient, a diagnosis of HCC was made based on the imaging findings, the presence of hepatitis B infection and elevated AFP. The BS findings and osteolytic lesions on ${ }^{18} \mathrm{~F}-\mathrm{FDGPET} / \mathrm{CT}$ suggested bone metastases. We doubt that the splenic lesions to be metastases from HCC for the patient underwent physical examination yearly and abdominal ultrasound didn't find any space-occupying lesion in the spleen before. Considering the advanced stage, surgical resection was not an option for this patient; instead, he underwent transcatheter arterial chemoembolization and local radiotherapy to reduce bone pain and prevent complications. The lesions in the liver and right seventh rib got slightly smaller. 3 month later, BS showed new anomalous radioactive concentration in T9, L2 and the left ninth rib (Figure 2); all the bone lesions which were hyperintense in hepatobiliary phase on Gd-EOB-DTPA enhanced MRI showed significant enlargement (Figure 3). The clinical follow-up confirmed that the bone lesions were metastases from HCC. 7 month after Gd-EOB-DTPA-enhanced MRI, the splenic nodules enlarged from 7-10 $\mathrm{mm}$ to $16-28 \mathrm{~mm}$, which confirmed the splenic nodules to be metastases from HCC (Figure 4).

\section{Discussion}

Gd-EOB-DTPA is a liver-specific MRI contrast agent that has up to $50 \%$ hepatobiliary excretion in the normal liver. Similar to a non-specific contrast agent, Gd-EOB-DTPA is distributed within the vascular and extravascular space during dynamic phases (arterial, portal venous and equilibrium phases) after intravenous injection and subsequently undergoes selective hepatobiliary excretion during the hepatobiliary phase [1]. During the hepatobiliary phase, liver lesions that have few normal hepatocytes, such as most HCCs, or lesions that are not of hepatocellular origin, appear as hypointense; whereas the background liver shows peak enhancement. This contrast agent improves HCCs detection, especially when lesions are small. However, 6\%-15\% of HCCs show Gd-EOB-DTPA uptake and appear as iso- or hyperintense relative to the background liver [2]. To the 

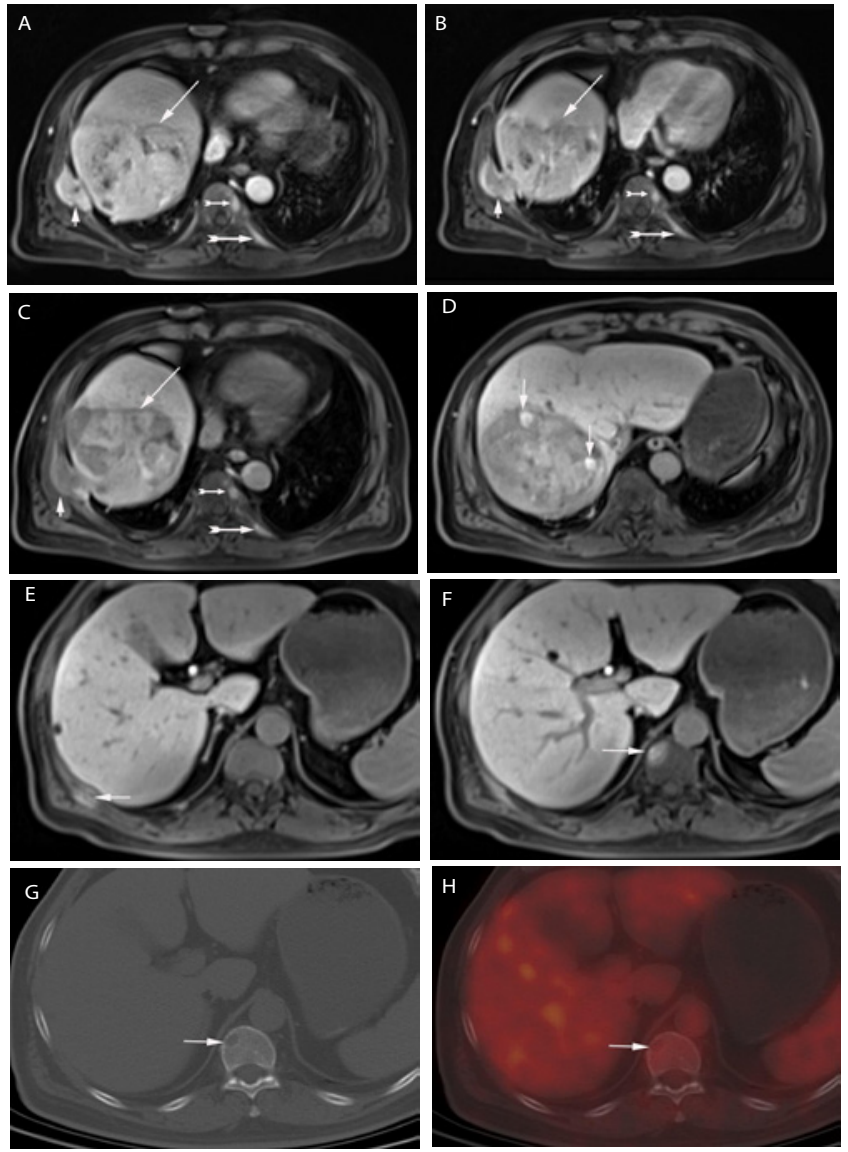

Figure 1: (A) (B) (C) The lesions in the liver (long arrow) and the right seventh rib (short arrow) appeared heterogeneously enhancement in the arterial phase, washout in the portal venous phase, and showed hypointense in the hepatobiliary phase. The lesions inT9 and left ninth rib (dovetail arrow) showed enhancement in the arterial phase and portal venous phase, with persistent enhancement in the hepatobiliary phase. (D) A few nodules (arrows) in the liver lesion were hyperintense in the hepatobiliary phase. (E) (F) The lesions in right tenth rib and T11 showed hyperintense in the hepatobiliary phase. (G)

(H) The osteolytic lesion in T11 didn't show elevated ${ }^{18}$ F-FDG uptake.

best of our knowledge, there has been only one case that described Gd-EOB-DTPA uptake in a metastatic adrenal tumour from HCC [3]. We report a case showing Gd-EOB-DTPA uptake in bone and splenic metastases from HCC.

The mechanism of Gd-EOB-DTPA uptake in HCCs and the characters of iso- or hyperintense HCCs have been gradually clarified. It is reported that the signal intensity of HCCs in hepatobiliary phase is related to the expression pattern of hepatocyte transporters. The major transporters of Gd-EOB-DTPA are organic anion transporter polypeptides OATP1B1/-1B3 (the uptake transporter) at the sinusoidal membrane, multi-drug resistance-associated proteins MRP2 (the excretion transporter) at the canalicular membrane and MRP3 (the excretion transporter) at the sinusoidal membrane. It was revealed that the enhancement ratio of HCCs in hepatobiliary phase is positively correlated with expression levels of OATP8 (synonymous with OATP1B3) and MRP3 [4], and OATP1B3 was confirmed as the most important uptake transporter of Gd-EOBDTPA $[5,6]$. Combined with OATP1B1 and/or OATP1B3 expression, two patterns of MRP2 expression contributed to high enhancement,

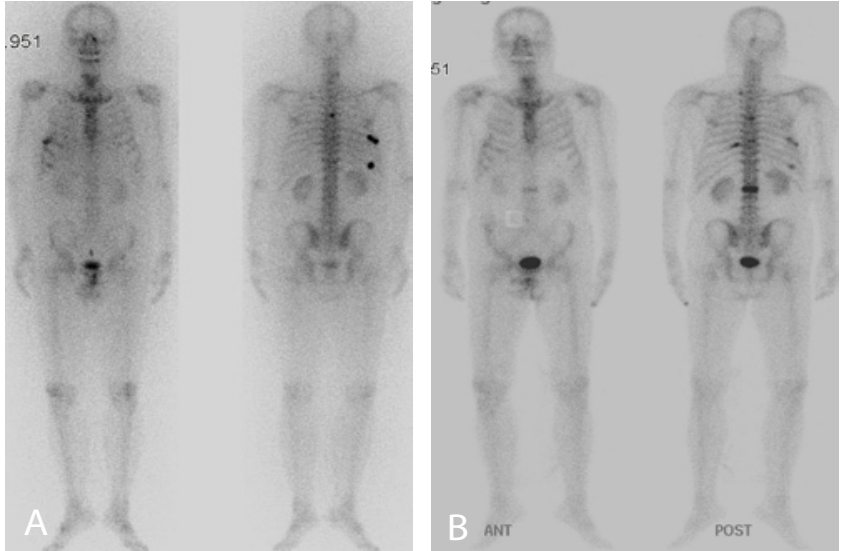

Figure 2: (A) Before treatment, BS showed anomalous radioactive concentration in $\mathrm{T} 5$, the right seventh and tenth ribs. (B) 3 month later, BS showed anomalous radioactive concentration in $\mathrm{T} 5, \mathrm{~T} 9, \mathrm{~L} 2$, the right seventh and tenth ribs, the left ninth rib.

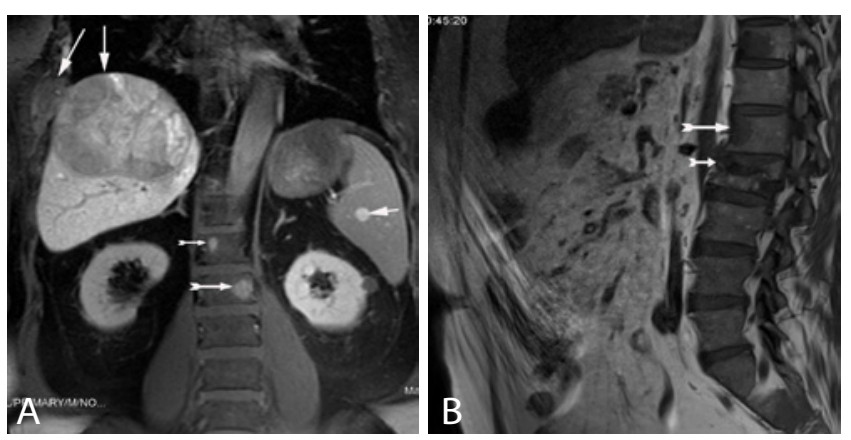

Figure 3: (A) In the hepatobiliary phase, the lesions in L1 and L2 (dovetail arrows) and spleen (short arrow) appeared hyperintense; the lesions in liver and right seventh rib showed hypointense (long arrow). (B) 3 months later, the lesion in L1 and L2 appeared significantly enlargement, with compression fracture of $L 2$.
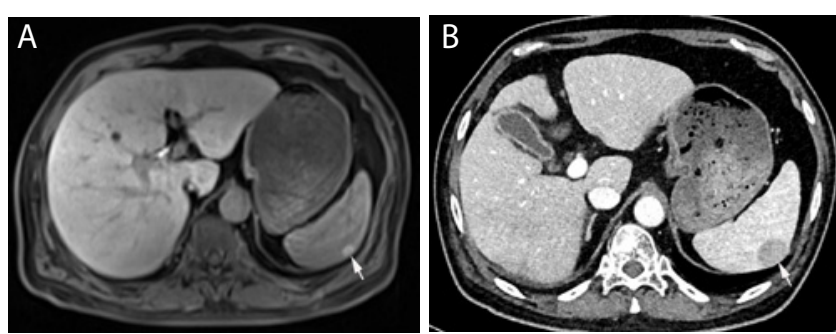

Figure 4: $(A)$ The splenic lesion was $10 \mathrm{~mm}$ and appeared hyperintense in hepatobiliary phase. (B) 7 month later, the splenic lesion enlarged to $28 \mathrm{~mm}$.

decreased expression at the canalicular membrane and increased expression at the luminal membrane of pseudo glands [7], which result in accumulation of Gd-EOB-DTPA in cytoplasm of tumour cells or lumina of pseudo glands. The iso- or hyperintense HCCs also have some histopathologic features, it was reportedthese HCCs tended to be of lower histologic grade $[2,6,8,9]$, a pseudo glandular proliferation pattern [4] and bile accumulation [4,7].

In our case, extrahepatic metastasis from HCC, except the lesion on the right seventh rib, appeared hyperintense in hepatobiliary phase; these findings were different from the signals in most part of the 
HCC lesions, but agree with the hyperintense nodules of HCC in the liver. It maybe that they have the same histological behavior with the hyperintense nodules of HCC in the liver, or the expression pattern of hepatocyte transporter changed resulted in more uptake or/and less excretion of Gd-EOB-DTPA, or it may be with lower histological grade or to be pseudo glandular proliferation pattern. Unfortunately, in this patient, the exact mechanism of Gd-EOB-DTPA uptake in the extrahepatic metastatic sites from HCC was not investigated. Nevertheless, we suppose that the extrahepatic lesions with GdEOB-DTPA uptake in the hepatobiliary phase indicate extrahepatic metastases from HCC. However, hyperintensity of a lesion in the hepatobiliary phase is not specific; some lesions, such as splenic hemangioma, may also appear as hyperintense in the hepatobiliary phase [10]. To differentiate between the two, hemangioma shows the classical light-bulb sign on T2WI, whereas HCC metastasis shows restricted diffusion-weighted imaging.

In our case, all the extrahepatic metastases which were hyperintense in hepatobiliary phase on Gd-EOB-DTPA enhanced MRI were not found elevated ${ }^{18} \mathrm{~F}-\mathrm{FDG}$ uptake on ${ }^{18} \mathrm{~F}-\mathrm{FDG}$ PET/CT, and only the lesions in T5 and the right tenth rib were found on BS. ${ }^{18} \mathrm{~F}-\mathrm{FDG}$ PET/CT have limitations of poor spatial resolution and low uptake of well differentiated HCCs. Poor spatial resolution results in low sensitivity for small lesions, the detection rate was only $13 \%$ of extrahepatic HCC metastases that are $\leq 10 \mathrm{~mm}$ [11]. The extrahepatic metastases which were hyperintense in hepatobiliary phase may be of lower histologic grade $[2,6,8,9]$ which resulted in low uptake of ${ }^{18} \mathrm{~F}$-FDG. Bone metastases from HCC are often osteolytic, this resulted in low sensitivity for most metastases from HCC on BS [12]. Our case may suggest that uptake of Gd-EOB-DTPA in extrahepatic lesions in hepatobiliary phase may indicate HCC extrahepatic metastasis, which may improve detection of extrahepatic metastasis from HCC.

\section{Conclusion}

We presented a case of bone and splenic metastases from HCC showing uptake of Gd-EOB-DTPA in hepatobiliary phase. Our case may suggest that uptake of Gd-EOB-DTPA in extrahepatic lesions in hepatobiliary phase may indicate HCC extrahepatic metastasis. Further studies on a large number of patients would be needed to validate our hypothesis.

\section{References}

1. Van Beers BE, Pastor CM, Hussain HK. Primovist, Eovist: what to expect?. J Hepatol. 2012; 57: 421-429

2. Kitao A, Matsui O, Yoneda N, Kozaka K, Kobayashi S, Koda W, et al. Hypervascular hepatocellular carcinoma: correlation between biologic features and signal intensity on gadoxetic acid-enhanced MR images. Radiology. 2012; 265: 780-789.

3. Arizono S, Isoda H, Hatano E, Togashi K. Uptake of gadolinium-ethoxybenzyldiethylenetriaminepentaacetic acid in metastatic adrenal tumour from hepatocellular carcinoma. Br J Radiol. 2011; 84: e182-e185.

4. Kitao A, Zen Y, Matsui O, Gabata T, Kobayashi S, Koda W, et al. Hepatocellular carcinoma: signal intensity at gadoxetic acid enhanced MR imaging - correlation with molecular transporters and histopathologic features. Radiology. 2010; 256: 817-826.

5. Ueno A, Masugi $Y$, Yamazaki K, Komuta M, Effendi $K$, Tanami $Y$, et al OATP1B3 expression is strongly associated with $\mathrm{Wnt} / \beta$-catenin signalling and represents the transporter of gadoxetic acid in hepatocellular carcinoma. J Hepatol. 2014; 61: 1080-1087.

6. Miura T, Ban D, Tanaka S, Mogushi K, Kudo A, Mastumura S, et al. Distinct clinicopathological phenotype of hepatocellular carcinoma with ethoxybenzylmagnetic resonance imaging hyperintensity: association with gene expression signature. Am J Surg. 2015; 210: 561-569.

7. Tsuboyama $\mathrm{T}$, Onishi $\mathrm{H}$, Kim $\mathrm{T}$, Akita $\mathrm{H}$, Hori $\mathrm{M}$, Tatsumi $\mathrm{M}$, et al. Hepatocellular carcinoma: hepatocyte-selective enhancement at gadoxetic acid-enhanced MR imaging--correlation with expression of sinusoidal and canalicular transporters and bile accumulation. Radiology. 2010; 255: 824833.

8. Choi JW, Lee JM, Kim SJ, Yoon JH, Baek JH, Han JK, et al. Hepatocellular carcinoma: imaging patterns on gadoxetic acid-enhanced MR images and their value as an imaging biomarker. Radiology. 2013; 267: 776-786.

9. Lee SA, Lee $\mathrm{CH}$, Jung WY, Lee J, Choi JW, Kim KA, et al. Paradoxical high signal intensity of hepatocellular carcinoma in the hepatobiliary phase of $\mathrm{Gd}$ EOB-DTPA enhanced MRI: initial experience. Magn Reson Imaging. 2011; 29: 83-90.

10. Makino I, Tajima $\mathrm{H}$, Kitagawa $\mathrm{H}$, Nakagawara $\mathrm{H}$, Ohta $\mathrm{T}$. A rare case of hemangiomatosis of the spleen and intrapancreatic accessory spleen. Abdom Imaging. 2014; 39: 1169-1174.

11. Sugiyama M, Sakahara H, Torizuka T, Kanno T, Nakamura F, Futatsubash $\mathrm{M}$, et al. 18F-FDG PET in the detection of extrahepatic metastases from hepatocellular carcinoma. J Gastroenterol. 2004; 39: 961-968.

12. Chen CY, Wu K, Lin WH, Lan TY, Wang SY, Sun JS, et al. High false negative rate of Tc-99m MDP whole-body bone scintigraphy in detecting skeletal metastases for patients with hepatoma. J Formos Med Assoc. 2012; 111:140-146.
Austin J Gastroenterol - Volume 4 Issue 1 - 2017

ISSN : 2381-9219 | www.austinpublishing group.com

Chen et al. () All rights are reserved
Citation: Zheng L, Zhao Y, Chen W and Tian R. Uptake of Gd-EOB-DTPA in Bone and Splenic Metastases from Hepatocellular Carcinoma: A Case Study. Austin J Gastroenterol. 2017; 4(1): 1075. 